\title{
Simposio \\ NueVos ESQUemas terapéUticos en PARASITOSIS
}

\section{O uso da terapêutica associado às medidas de saneamento básico no controle da Schistosoma mansoni no Brasil}

\author{
Naftale Katz \\ Fundação Oswaldo Cruz, Belo Horizonte, Brasil
}

Serão apresentados os resultados obtidos em duas áreas endêmicas de Schistosoma mansoni em Minas Gerais, Brasil. Nestas áreas, os trabalhos iniciaram-se na década de 1980 e tiveram como medidas de controle o uso da quimioterapia específica (oxmniquine ou prazinquantel), para tratamento dos casos positivos, diagnósticos pelo exame parasitológico das fezes (método de KatoKatz), associados às medidas de saneamento básico que foram introdução de água encanada nas residências e de privadas.

O re-exame das populações de residentes destas duas áreas endêmicas, após 25 anos dos programas de controle, mostrou que foi possível diminuir a prevalência da esquistossomose de $70 \%$ inicialmente para menos de $2 \%$ e de $40 \%$ também para o mesmo percentual da primeira. Em ambos, não houve aparecimento de novos casos de forma hepatoesplênica que era em torno de $10 \%$ na primeira área, no início do programa. Serão ainda apresentados os dados referentes ao saneamento básico no Brasil.

O autor sugere que estes exemplos sejam multiplicados em todo o país, onde deverá ser dada prioridade para o saneamento básico que em associação com medida de tratamento específico poderão fazer o controle da esquistossomose.

\section{Nuevos esquemas terapéuticos en teniasis y cisticercosis debidas a Taenia solium}

\author{
Ana Flisser ${ }^{1}$, Agnés Fleury ${ }^{2}$ \\ 1 Departamento de Microbiología y Parasitología, Facultad de Medicina, Universidad Nacional \\ Autónoma de México, México, D.F., México \\ 2 Instituto de Investigaciones Biomédicas, UNAM, e Instituto Nacional de Neurología y Neurocirugía \\ "Manuel Velasco Suarez", México, D.F., México
}

\section{El parásito y su ciclo de vida}

Taenia solium tiene tres estadios de desarrollo: el gusano adulto, el huevo y la forma larvaria llamada cisticerco. El parásito adulto, o tenia, es un gusano plano, angosto, delgado y blanquecino que habita de manera natural sólo en el intestino delgado de los seres humanos. La tenia mide entre 2 y $4 \mathrm{~m}$ de largo, está formada por un escólex que tiene una doble corona de ganchos y cuatro ventosas y, en conjunto se llama róstelo, seguido por el estróbilo, que está formado por cientos de segmentos, llamados proglótidos, que pueden ser inmaduros, maduros o grávidos, los inmaduros están al principio; los maduros, son hermafroditas y los grávidos, que están al final, contienen alrededor de 60.000 huevos cada uno y se eliminan con las heces. Los huevos son microscópicos, cada uno contiene una oncosfera o embrión llamado hexacanto por tener seis ganchos. El cisticerco, que se desarrolla en el músculo esquelético, el cerebro y el ojo del cerdo, es una vesícula transparente, mide $1 \mathrm{a} 2 \mathrm{~cm}$, está lleno de fluido y contiene al escólex invaginado, que es el mismo de la tenia.

La tenia se desarrolla en una persona después de ingerir cisticercos al comer carne de cerdo insuficientemente cocinada. Por estímulo de las sales biliares, el escólex evagina, su róstelo se adhiere a la mucosa del intestino delgado y, después de 3 a 4 meses de desarrollo, la tenia se vuelve grávida y elimina huevos. Cuando los seres humanos o los cerdos ingieren huevos, los jugos gástricos e intestinales activan las oncosferas o 
larvas recién nacidas, las que atraviesan la mucosa intestinal, circulan y se transforman en cisticercos en el músculo y el sistema nervioso central, y son visibles después de 3 a 4 meses. En los seres humanos los cisticercos causan neurocisticercosis que es una enfermedad de importancia en salud pública en muchos países de América Latina, África subsahariana y Asia y se asocia, principalmente, con epilepsia. Es importante señalar que las personas con cisticercosis no participan en el ciclo de vida porque no transmiten la infección, en cambio aquellas portadoras de la tenia intestinal, son el principal factor de riesgo para generar casos de cisticercosis humana y porcina.

\section{Tratamiento de la teniasis}

Al principio de la era cristiana, algunos autores árabes tales como Serapio llamaron a las tenias "cucurbitini" no solamente por su semejanza con pepitas de calabaza sino porque éstas son uno de los remedios más antiguos contra la teniasis. Durante el decenio de 1950, los medicamentos empleados para eliminar a la tenia eran: mecaprina, compuestos de estaño, paromomicina, derivados de la acridina, diclorofén, triclorofén, bitionol y extractos del helecho macho Aspidium.

Selogróungranavanceenlaquimioterapiacuando se introdujo la niclosamida en 1960, una cloronitro-fenil-cloro-salicilamida, y, más recientemente, el prazicuantel, una isoquinolina-pirazina acilada sintética. El efecto de estos fármacos es diverso, pero el conocimiento preciso de su modo de acción es escaso. El prazicuantel, la paromomicina y los compuestos de estaño desgarran el tegumento del gusano. El prazicuantel también interfiere con los canales de calcio paralizando al parásito, al igual que el extracto de helecho macho. La niclosamida desacopla la fosforilación oxidativa y, por lo tanto, inhibe la captación de oxígeno y glucosa.

No se conoce el modo de acción de la semilla de calabaza, de los derivados de acridina y de la paromomicina; esta última es un antibiótico del que se descubrió suacción antihelmíntica por casualidad o serendipity. Indudablemente, el prazicuantel y la niclosamida son los medicamentos de elección para el tratamiento de la teniasis y su eficacia ha sido exhaustivamente investigada; se administran como dosis única oral en la mañana antes de los alimentos y puede darse una hora después una purga salina.

El prazicuantel se administra en una dosis de $10 \mathrm{mg} / \mathrm{kg}$ y produce cerca de $100 \%$ de curación, con pocos efectos colaterales tales como dolor abdominal o de ligero y transitorio dolor de cabeza y, en casos raros, mareos y erupciones en piel. La niclosamida se administra en una dosis total de 2 g para adultos en 4 tabletas de $500 \mathrm{mg}$. Un solo tratamiento es efectivo en $80 \%$ a $90 \%$ de los casos. La niclosamida, a diferencia del prazicuantel, no tiene contraindicaciones y puede emplearse durante el embarazo y en niños.

También se puede emplear la paromomicina, cuando los otros dos fármacos no están disponibles, en una dosis única de $75 \mathrm{mg} / \mathrm{kg}$ con un máximo de $4 \mathrm{~g}$, después de la comida. Se pueden presentar efectos secundarios leves, principalmente diarrea y vómito.

Aún se utilizan los derivados de la acridina (quinacrina) en los países asiáticos, a pesar del riesgo de baja tolerancia y sobredosis; en China se emplean las nueces de areca con alta eficacia.

Por último, el albendazol, un derivado benzimidazólico, se emplea en casos de parasitosis múltiples por su efectividad contra cestodos y nematodos. La dosis recomendada para el tratamiento de teniasis es de $6,6 \mathrm{mg} / \mathrm{kg}$; una dosis única de $400 \mathrm{mg}$ produjo $65 \%$ de curación contra teniasis, mientras que 3 días de dosificación aumentó la curación al $86 \%$. El albendazol no debe usarse en niños menores de 2 años o durante el embarazo, debido a los efectos embriotóxicos y teratogénicos que se han observado en animales experimentales.

La quimioterapia se puede considerar exitosa si no reaparecen proglótidos en la materia fecal 4 meses después del tratamiento. Si es necesario, se puede repetir la administración de la droga algunas semanas después del primer tratamiento y también si vuelven a desalojarse proglótidos 3 a 4 meses después.

El cuadro que se presenta en la parte superior de la página siguiente es una recopilación de los resultados de los tratamientos de teniasis con diversos medicamentos cestocidas y una purga, que se administró con base en el diagnóstico de laboratorio confirmatorio por examen coprológico, historia de haber arrojado proglótidos o ensayo inmunoenzimático (ELISA) y muestra eficacias variables (Flisser, 2006).

\section{Tratamiento de la neurocisticercosis}

Una de las principales características de la neurocisticercosis es su gran heterogeneidad tanto a nivel clínico como radiológico. Los pacientes pueden presentar cuadros benignos (cefalea) pero también cuadros serios, pudiendo llegar a poner la vida del paciente en peligro (epilepsia, hipertensión intracraneana). Esta variabilidad 


\begin{tabular}{llrlll}
\hline Casos & Diagnóstico & Tenias & Tratamiento & Purga & Eficacia \\
\hline 85 & Coprológico & 60 & Pepitas de calabaza & Salina antes y después & No determinado \\
7 & Coprológico & 2 & Salicilamida & Purga salina 1-2 h después & $70 \%$ \\
4 & Historia & 3 & Prazicuantel & Purga salina 1-2 h después & $60 \%$ \\
5 & Historia & 3 & Prazicuantel & Purga salina 1-2 h después & $33 \%$ \\
36 & ELISA & 13 & Prazicuantel & Purga salina 1-2 h después & $40 \%$ \\
5 & ELISA & 2 & Niclosamida & Aceite de ricino en comunidad & $41 \%$ \\
17 & Coprológico & 7 & Niclosamida & Polietilén-glicol 4 l, comunidad & $69 \%$ \\
36 & Coprológico & 25 & Niclosamida & Aceite de ricino en hospital & $31 \%$ \\
29 & Coprológico & 9 & Niclosamida & Polietilén-glicol 4 l, en hospital & $53 \%$ \\
32 & Coprológico & 17 & Niclosamida & Purga salina 1-2 h después & $78 \%$ \\
9 & ELISA & 7 & Niclosamida & Purga salina 1-2 h después & $50 \%$ \\
2 & Coprológico & 1 & Niclosamida & Purga salina 1-2 h después & $57 \%$ \\
12 & Coprológico & 7 & Niclosamida & Purga salina 1-2 h después & $0 \%$ \\
21 & ELISA & 0 & Prazicuantel & Purga salina 1-2 h después & $0 \%$ \\
20 & ELISA & 0 & Prazicuantel & & \\
\hline
\end{tabular}

clínica se relaciona con una heterogeneidad del número, la localización y el estado evolutivo de los parásitos, así como de la intensidad de la reacción inflamatoria generada.

La neurocisticercosis "benigna" se caracteriza por tener localización parenquimatosa del parásito, enfermedad poco inflamatoria y paucisintomática, mientras que la "maligna" es de localización ventricular o en el espacio subaracnoideo de la base, es inflamatoria y presenta sintomatología potencialmente grave. El pronóstico de los pacientes y el tratamiento administrado son diferentes en estos dos grupos, aunque los objetivos del tratamiento sean en todos los casos: destruir el parásito, controlar la reacción inflamatoria y controlar los síntomas generados por la presencia del parásito.

Para eliminar el parásito existen de dos fármacos cestocidas:elalbendazol (utilizadoporestepropósito desde 1987) y el prazicuantel (utilizado desde 1979). Tanto el uno como el otro han demostrado ser muy eficaces en los parásitos de localización parenquimatosa, logrando su desaparición o calcificación, y la reducción del número de crisis epilépticas. Debido a una ligera eficacia mayor del albendazol sobre el prazicuantel, así como un precio menor, el albendazol se considera el tratamiento de elección. Cuando el cisticerco se localiza en el espacio subaracnoideo de las cisternas de la base o en el sistema ventricular, la eficiencia de estos fármacos es menor. En estos pacientes, a pesar de utilizar una dosis doble de albendazol es muy frecuente que sea necesario administrar varios ciclos de albendazol durante varios años antes de lograr la completa desaparición de los parásitos.

Para el control de la reacción inflamatoria, indispensable en los casos de neurocisticercosis "maligna", los fármacos más frecuentemente utilizados son los corticoides. Aunque han demostrado reducir la morbilidad y la mortalidad de estos pacientes, su administración, a veces crónica, se asocia con efectos indeseables potencialmente graves y su eficiencia en la reducción de la inflamación es a veces parcial. Con relación al control sintomático, la acción quizá más relevante es colocar quirúrgicamente una derivación ventrículoperitoneal. Este acto sencillo permite que se remita la hipertensión intracraneana frecuente en la forma "maligna" de neurocisticercosis, aunque si la válvula no es de buena calidad, es necesario reemplazarla con cierta periodicidad. Además, frecuentemente es necesario hacer uso de antiepilépticos y analgésicos.

Enresumen, aunque hahabidograndes adelantos, en ciertos pacientes los fármacos cestocidas no logran controlar la infección y los corticoides no pueden regular la inflamación asociada. Ya están en curso algunas investigaciones para tratar de encontrar nuevas opciones cestocidas y antiinflamatorias.

\section{Bibliografía}

1. Cárdenas G, Carrillo-Mezo R, Jung H, Sciutto E, Hernández JL, Fleury A. Subarachnoidal neurocysticercosis non-responsive to cysticidal drugs: a case series. BMC Neurol. 2010;10:16.

2. Del Brutto OH, Roos KL, Coffey CS, García HH. Metaanalysis: cysticidal drugs for neurocysticercosis: albendazole and praziquantel. Ann Intern Med. 2006;145:43-51.

3. Fan PC. Taiwan taenia and taeniasis. Parasitol Today. 1988;4,86-8.

4. Fleury A, Carrillo-Mezo R, Flisser A, Sciutto E, Corona T. Subarachnoid basal neurocysticercosis: a focus on the most severe form of the disease. Expert Rev Anti 
Infect Ther. 2011;9:123-33.

5. Fleury A, Escobar A, Fragoso G, Sciutto E, Larralde C. Clinical heterogeneity of human neurocysticercosis results from complex interactions among parasite, host and environmental factors. Trans R Soc Trop Med Hyg. 2010;104:243-50.

6. Flisser A, Viniegra AE, Aguilar-Vega L, GarzaRodríguez A, Maravilla P, Ávila G. Portrait of human tapeworms. J. Parasitol. 2004.90:914-6.

7. Flisser A. Where are the tapeworms? Parasitol Intl. 2006;55; S117-20.

8. García HH, Evans CAW, Nash TE, Takayanagui OM, White AC, Botero D, Rajshekhar V, et al. Current consensus guidelines for treatment of neurocysticercosis. Clin Microbiol Rev. 2002;15:74756.

9. García HH, González AE, Evans CA, Gilman RH.
Taenia solium cysticercosis. Lancet. 2003;362:547-56.

10. Groll E. Praziquantel. Adv Pharmacol Chemother. 1984 20,219-38.

11. Grove DI. A history of human helminthology. Oxon, UK: CAB International. 1990. p.355-83.

12. Jung $H$, Cárdenas $G$, Sciutto E, Fleury A. Medical treatment for neurocysticercosis: drugs, indications and perspectives. Curr Top Med Chem. 2008;8:42433.

13. Pawlowski ZS, Schultz MG. Taeniasis and cysticercosis. Adv Parasitol. 1972;10,269-343.

14. Sharma S, Dubery SK, lyer RN. Chemotherapy of cestode infections. Prog Drug Res. 1980;24:219-66.

15. Sotelo J, Flisser A. Neurocysticercosis: practical treatment guidelines. CNS Drugs. 1997;7:17-25.

\section{Fascioliasis}

César Náquira

Universidad Nacional Mayor de San Marcos, Lima, Perú

\section{Esquema terapéutico en humanos}

Se deben considerar las formas clínicas: invasiva o aguda, crónica o de estado y extrahepática.

\section{Fasciolicidas}

De uso discontinuado: por nula o escasa elaboración

Emetina: $1 \mathrm{mg} / \mathrm{kg}$ diario por 10 días, inyectable

Dehidroemetina: igual dosis, por vía oral

Bithionol: $30 \mathrm{mg} / \mathrm{kg}$ diario por 20 días, por vía oral, con un día intermedio

\section{De uso actual:}

Droga de elección: triclabendazol: 10 a $12 \mathrm{mg} /$ $\mathrm{kg}$, dosis única o dos dosis, por vía oral después del desayuno o alimento, con un día intermedio. Recomendado universalmente y por su ventaja sobre los medicamentos discontinuados.

Nitazoxanida: $500 \mathrm{mg}$ dos veces al día por 6 días; de uso, en algunos países, con resultados diversos.

Prazicuantel: se considera ineficaz para esta parasitosis.

Control del tratamiento: hasta los 90 días después del tratamiento

Examen de heces, buscando huevos de Fasciola hepatica o coproantígenos

Cuerda encapsulada buscando huevos de $F$. hepatica en el jugo duodenal

Observación del Western Blot que desaparece después de meses

\section{Formas extrahepáticas}

Pueden requerir tratamiento quirúrgico.

\section{Resistencia}

No se presenta en medicina humana con triclabendazol, a diferencia de lo que sucede en medicina veterinaria.

\section{Tratamientos comunitarios}

Se ha usado dosis única de triclabendazol asociado a programas de control de la fascioliasis animal en países con alta prevalencia.

\section{Lecturas recomendadas}

1. Apt W, Aguilera X, Vega F, Miranda C, Zulantay I, Pérez C, Gabor M, Apt P. Treatment of human chronic fascioliasis with triclabendazole: drug efficacy and serologic response. Am J Trop Med Hyg. 1995;52:532-5.

2. Lecaillon JB, Godbillon J, Campestrini J, Naquira C, Miranda L, Pacheco R, Mull R, Poltera A,A, Effect of food on the bioavailability of triclabendazole in patients with fasciliasis. Br J Clin Pharmacol. 1998;45:601-4.

3. Rossignol JF, Abaza H, Friedman H. Successful treatment of fascioliasis with nitazoxanida. Trans $\mathrm{R}$ Soc Trop Med Hyg. 1998;92:103-4.

4. Talaie H, Habib E, Yadegarina D, Nava-Ocampo A, Massoud J, Azmuudeh M, Mas-Coma S. Randomized trial of a single, double and triple dose of $10 \mathrm{mg} / \mathrm{kg}$ of a human formulation of triclabendazole in patients with fascioliasis. Clin Exp Pharmacol Physiol. 2004;31:777-82. 


\title{
Tratamiento de la enfermedad de Chagas, estado actual
}

\author{
Werner Apt \\ Laboratorio de Parasitología Básico-Clínico, Programa de Biología Celular y Molecular, \\ Instituto de Ciencias Biomédicas, Facultad de Medicina, Universidad de Chile, Santiago, Chile
}

La enfermedad de Chagas debe ser tratada en todos sus períodos: agudo, crónico indeterminado y crónico determinado inicial e intermedio por el hecho de que el ADN del parásito ha sido demostrado mediante PCR en casos crónicos en los que la microscopia óptica y electrónica no los pesquisa. Los únicos casos que no deben recibir terapia son el core bovis y las cardiomiopatías con insuficiencia cardiaca crónica terminal.

Los fármacos aceptados por razones éticas $y$ de eficacia en el hombre son nifurtimox y el benznidazol. Ambos originan efectos secundarios hasta en $30 \%$ de los casos y la terapia debe administrarse, por lo menos, 30 a 60 días. Por los efectos secundarios de los fármacos, los pacientes deben ser controlados clínicamente, mediante hemograma y perfil hepático antes, durante y después del tratamiento. Otros fármacos útiles son el itraconazol y el posaconazol, ambos compuestos triazólicos. El posaconazol podría ser el fármaco del futuro si las investigaciones -que recién se inician- en casos humanos demuestran su efectividad.

En la actualidad no existe un criterio de curación de los casos crónicos ya que la serología aunque decreciente en los casos tratados puede persistir positiva por más de 20 años, no obstante, los pacientes no presentan tripanosomas con las técnicas actualmente en uso: xenodiagnóstico, hemocultivo, PCR y qPCR. En los casos agudos $70 \%$ de curación se obtiene con nifurtimox y $75 \%$ con benznidazol. En los casos congénitos, 100\% de curación con ambos fármacos si la terapia se efectúa dentro del primer año de vida. En los casos crónicos adquiridos, 10 a 30\% de curación se obtiene con benznidazol y $20 \%$ de curación, mejoría de la cardiopatía inicial en 50\% y franca disminución del desarrollo de cardiopatía en los pacientes con el período crónico indeterminado tratados con itraconazol frente a controles no tratados.

El tratamiento modifica la evolución natural de la enfermedad y como el número de pacientes con enfermedad de Chagas es de alrededor de 8 a 10 millones la terapia soluciona un problema de salud pública.

\section{Referencias}

1. Apt W. Estado actual del tratamiento de la enfermedad de Chagas. Rev Méd Chile. 2011;139:247-57.

2. Apt W. Current and developing therapeutic agents in the treatment of Chagas disease. Drug Des Devel Ther. 2010;4:243-53.

3. Apt W. Treatment of Chagas disease. En: Telleria $\mathrm{J}$, Tibayrenc M, editors. American trypanosomiasis. Chagas' disease. New York: Elsevier; 2010. p 80925.

4. Pinto Dias J. Tratamiento etiológico de la enfermedad de Chagas. XII Reunión de la Comisión Intergubernamental del Cono Sur para la eliminación de Triatomo infestans y la interrupción de la transmisión transfusional de la Tripanosomiasis Americana (INCOST/Chagas). Washington, D.C.: PAHO; 2004. p. 129-34.

5. Urbina J. New chemotherapeutic approaches for the treatment of Chagas disease (American trypanosomiasis). Expert Opin Ther Pat. 2003;13:661-9.

6. Viotti R, Vigliano C, Lococo B, Alvarez M, Petti M, Bertocchi G, Armenti A. Side effects of benznidazole as treatment in chronic Chagas disease: fears and realities. Expert Rev Anti Infect Ther. 2009;7:157-63.

\section{Tratamiento de las helmintiasis intestinales transmitidas por el suelo}

\author{
David Botero \\ Instituto Colombiano de Medicina Tropical, Medellín, Colombia
}

\section{Antihelmínticos}

Benzimidazoles: albendazol, mebendazol y flubendazol. El primero, $400 \mathrm{mg}$, y los otros dos, $500 \mathrm{mg}$, dosis única para ascariasis y por 3 días para tricocefalosis y uncinariasis.
Pirantel-oxantel: $10 \mathrm{mg}$ de cada componente, en dosis única para ascariasis y por 3 días para tricocefalosis y uncinariasis.

Piperacina, $50 \mathrm{mg} / \mathrm{kg}$ por 5 días para obstrucción intestinal por ascariasis 
Resistencia: no se presenta en medicina humana a diferencia de lo que sucede en medicina veterinaria.

Tratamientos comunitarios: principalmente en ascariasis en comunidades con $20 \%$ o más de prevalencia de esta parasitosis. Consiste en la administración de una dosis única, generalmente de un benzimidazol, una a dos veces por año, a los escolares, durante 5 o más años.

Es preferible hacerlo asociado a campañas de salud y acompañado de medidas higiénicas y de saneamiento ambiental. Se reducen parcialmente tricocefalosis y uncinariasis. Es recomendado por la Organización Mundial de la Salud (OMS) y por el Banco Mundial, por su buen costo-beneficio.

Uso durante el embarazo: en lo posible, evitarlo en el primer trimestre, aunque la OMS no refiere anormalidades en niños nacidos de madres que lo recibieron en el primer trimestre.

\section{Lecturas recomendadas}

1. Botero, D, Retrepo M. Parasitosis humanas. Quinta edición. Medellín: Editorial CIB: 2010.

2. Flisser A, Valdespino JL, et al. Using national health weeks to deliver deworming to children: lessons from México. J Epidemiol Community Health. 2008;62:314-7.

3. Organización Mundial de la Salud. Medicamentos utilizados en las enfermedades parasitarias. Segunda edición., Ginebra: OMS; 1996.

4. OrganizaciónPanamericanadelaSalud.Lineamientos para la evaluación de las geohelmintiasis y la esquistosomiasis a nivel de la comunidad. Guía para el manejo de las actividades de control. Washington, D.C., OPS; 1998.

\title{
Tratamiento de las parasitosis intestinales en pacientes inmunocompetentes e inmunodeprimidos
}

\author{
Benjamin Cimerman
}

Instituto de Ensino e Pesquisa do Hospital Albert Einstein, São Paulo, Brasil

Las parasitosis intestinales constituyen un problema de salud pública en los países en desarrollo que afectan primordialmente las comunidades rurales y marginales en América Latina.

La identificación adecuada y el tratamiento de las parasitosis intestinales pueden resultar en beneficio para los pacientes y la salud pública.

En los últimos años, la terapéutica de las parasitosis en pacientes inmunocompetentes e inmunodeprimidos ha ido progresando con nuevos fármacos.

Se discute una actualización de la farmacoterapia disponible en la actualidad contra la giardiasis, la criptosporidiosis, isosporosis, microsporidiosis, blastocistosis y estrongiloidiasis.

\section{Referencias}

1. Cimerman S, Cimerman B, Lewi DS. Enteric parasites and AIDS. Sao Paulo Med J. 1999;117:266-73.

2. Escobedo AA, Almirall $P$, Alfonso M, Avila I, Cimerman S, Salazar Y, Dawkins IV, García RM. Caregiver perspectives for the prevention, diagnosis and treatment of childhood giardiasis in Havana City, Cuba. A qualitative study. Acta Trop. 2011 May 4. [Epub ahead of print]

3. Muñoz $P$, Valerio $M$, Puga $D$, Bouza E. Parasitic infections in solid organ transplant recipients. Infect Dis Clin North Am. 2010;24:461-95. 